\title{
Left basilar VATS segmentectomy for intra lobar pulmonary sequestration using indocyanine green for identification of the intersegmental plane: a case report
}

\author{
René Horsleben Petersen^ \\ Department of Cardiothoracic Surgery, Copenhagen University Hospital, Copenhagen, Denmark \\ Correspondence to: Professor René Horsleben Petersen, MD, PhD. Department of Cardiothoracic Surgery, Copenhagen University Hospital, \\ Copenhagen, Denmark. Email: rene.horsleben.petersen@regionh.dk.
}

\begin{abstract}
Pulmonary sequestration is a rare congenital abnormality that requires surgical resection if symptomatic. The pulmonary sequestration can be extra lobar with a separate visceral pleura, artery and venous drainage or intra lobar contained within the visceral pleura of the lung with a separate artery and venous drainage to the pulmonary vein. Rarely the entire lobe is affected in intra lobar sequestration and segmentectomy should be considered rather than lobectomy. A case of an intra lobar sequestration in the left lower lobe with a feeding artery coming from the descending thoracic aorta is presented. The patient was a young woman, who had experienced recurrent pneumonia for one year. A left video-assisted thoracoscopic surgery (VATS) basilar segmentectomy was performed using a standardized anterior threeport approach. Due to the recurrent infections, the hilar lymph nodes were fibrotic, and dissection was performed using an advanced energy system. Identification of the intersegmental plane may be challenging in segmentectomies. Most surgeons use an inflation-deflation technique. In this case identification was performed using a near-infra red camera and intravenous injection of indocyanine green. Identification was successful. The postoperative course was uneventful with a length of stay of two days. On follow up 6 months postoperatively, the patient was without any symptoms.
\end{abstract}

Keywords: segmentectomy; pulmonary sequestration; intersegmental planes; indocyanine green; case report

Received: 06 May 2021; Accepted: 24 May 2021; Published online: 17 June 2021.

doi: 10.21037 /jovs-21-21

View this article at: http://dx.doi.org/10.21037/jovs-21-21

\section{Introduction}

Pulmonary sequestration is a rare congenital anomaly of the primitive foregut, where a part of the lung is dysplastic without communication to the bronchial tree and with systemic vascular supply. The condition was firstly described in 1946 by Pryce (1). Pulmonary sequestration can be extra lobar with a separate visceral pleura, artery and venous drainage or intra lobar within the visceral pleura of the lung, separate artery and venous drainage to the pulmonary vein. The artery usually arises from the descending thoracic aorta or the abdominal artery, but can arise from other systemic arteries such as the celiac trunk, splenic artery or intercostal arteries. Two thirds of the intra lobar sequestrations are found in the basilar segments of the left lower lobe. Approximately $60 \%$ are diagnosed in adults. The most common symptom is recurrent pneumonia. Other symptoms may be back pain, persistent cough and hemoptysis (2). Occasionally the sequestration is an incidental finding on a computerized tomography (CT) scan. When symptomatic, a lobectomy is usually scheduled, however since this a benign disease, it is often not the entire lobe that is affected and a segmentectomy should be considered (3). I present the following case in accordance

^ ORCID: 0000-0002-3586-1869. 

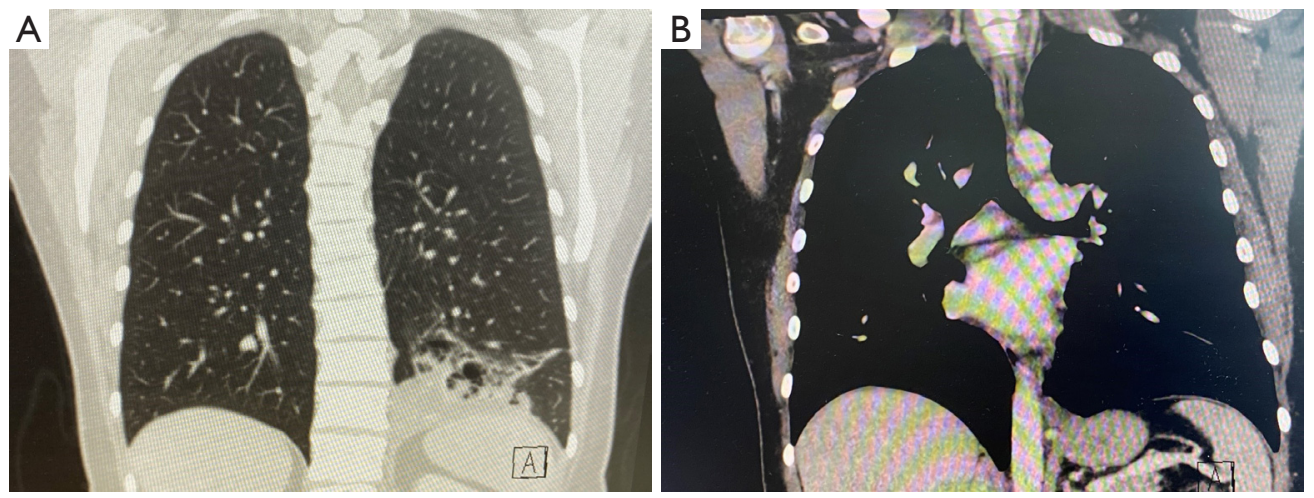

Figure 1 Preoperative CT showing an intra lobar sequestration in the left lower lobe. (A) Coronal view, lung window. (B) Coronal view, mediastinal window.

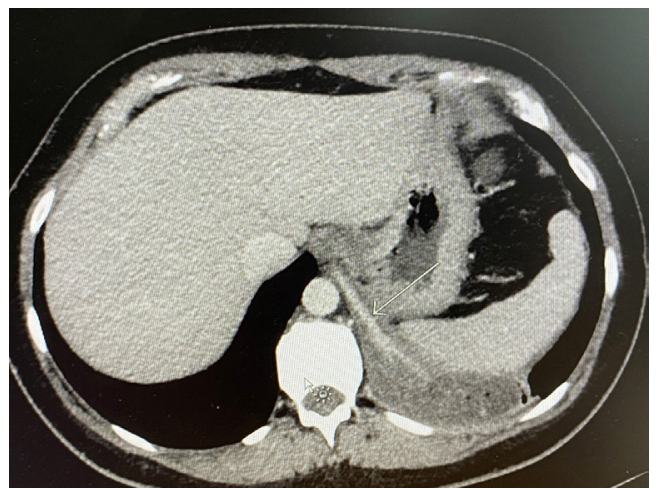

Figure 2 Preoperative CT scan with contrast showing an intra lobar sequestration in the left lower lobe and the arrow marks the feeding artery from the descending aorta.

with the CARE reporting checklist (available at https://jovs. amegroups.com/article/view/10.21037/jovs-21-21/rc).

\section{Case presentation}

This was a 34-year-old woman presenting with recurrent pneumonia during one-year, persistent cough and two incidents of hemoptysis. Firstly, a chest X-ray was performed, then a CT scan using intravenous contrast revealing an intra lobar sequestration affecting the basilar segments of the left lower lobe (Figure 1A,1B). The systemic arterial blood supply was arising from the descending aorta at the level of the diaphragm, Figure 2. The patient was a former smoker (5 pack-years) and in good performance status with a FEV1 2.65 (100\%) and a DLCO 88\%.

All procedures performed in this study were in accordance with the ethical standards of the institutional and/or national research committee(s) and with the Helsinki Declaration (as revised in 2013). Written informed consent was obtained from the patient for publication of this manuscript and the accompany images and video. A copy of the written consent is available for review by the editorial office of this journal.

\section{Pre-operative preparation}

For pulmonary sequestrations localization of the of the arterial supply is crucial and a preoperative CT scan with contrast is mandatory. For segmentectomies $3 \mathrm{D}$ CT reconstruction is a helpful tool to localize the vascular supply and the bronchial anatomy at the segmental level, where many anatomical variations may be present. In this case only a CT with contrast was available. Based on the CT findings of arterial blood supply arising from the descending aorta and that the sequestration did not include segment 6, a basilar segmentectomy was scheduled.

\section{Procedure}

A standardized anterior three-port video-assisted thoracoscopic surgery (VATS) approach as previously described was used for this case $(4,5)$. The video of the case is attached (Video 1). The patient was positioned in the lateral decubitus position and the operation table bend at the level of the xiphoid to make the intercostal spaces wider. A double lumen camera tube (Vivasight, Ambu, Denmark) secured left lung isolation. A 3-4 cm anterior utility port was created in fourth intercostal space anterior to the latissimus dorsi muscle and a wound protector (Surgi, Sleeve, Medtronic, USA) was inserted. The thoracic cavity was inspected, and 


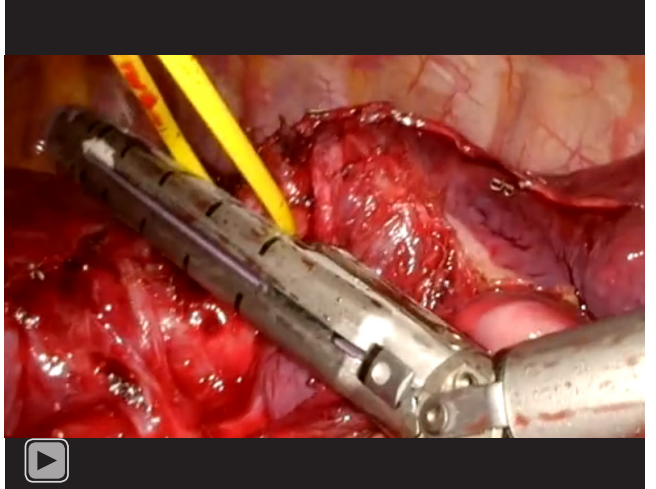

Video 1 Left basilar VATS segmentectomy for intra lobar pulmonary sequestration using indocyanine green for identification of the intersegmental plane.

another two inferior ports were placed at the level of the diaphragm. A 30-degree, $10 \mathrm{~mm}, 3 \mathrm{D}$ thoracoscope (Endoeye, Olympus, Japan) was inserted in the lower anterior port. Then $20 \mathrm{~mL}$ of Marcaine $0.5 \%$ was applied as a single shot paravertebral in intercostal space 2-10 (5). Adhesions were removed and the inferior ligament were carefully dissected with Ligasure (Medtronic) to localize the feeding artery to the sequestration. The feeding artery was one $\mathrm{cm}$ in diameter and an endostapler, purple cartilage (Signia, Medtronic) was used to divide the artery, keeping the stump long enough to stay within the thoracic cavity. The hilar lymph nodes were affected by the recurrent infections and dissection was performed using Ligasure. The inferior pulmonary vein was dissected, and the dissection was continued to the hilar part of pleura to localize the 6 . segmental vein. The branches to the basilar segments was isolated, slinged with a vessel loop and divided with an endostapler using a tan cartilage. Thereafter dissection was continued in the anterior part of the oblique fissure. The pulmonary artery was localized, and a "tunnel" was created over the artery and the lung parenchyma was divided using purple cartilages. The basilar arteries were dissected, slinged and stapled using a tan cartilage. Bronchus for the basilar segments were dissected and stapled with a purple cartilage. The left lung was inflated to control for the 6. segmental bronchus and the basilar hilar structures were mobilized distally with Ligasure to avoid obstruction of hilar structures during division of the intersegmental plane. To identify the intersegmental plane, $5 \mathrm{mg}$ of Indocyanine green was injected by the anesthesiologist and the 3D camera was replaced by a near infrared camera (Elevision, Medtronic) with a fused near infrared/HD visualization mode. The 6 . segment colored green due to the intact blood supply and the reaming segments were uncolored. The intersegmental plane was marked with electrocoagulation. The $3 \mathrm{D}$ camera is introduced again, and the intersegmental plane was divided using black cartilages. The basilar segments were removed in an endobag. A water test was performed under insufflation of the left lung to control for air leak and insufflation of the remaining segment. Vessels and bronchus for the 6 . segment was inspected for potential obstruction. A single chest drain $\mathrm{CH} 24$ was inserted in the camera port and an intercostal catheter was applied at the chest drain site as a continuous block of the intercostal nerve connected to a pump with $0.25 \%$ Marcaine $6 \mathrm{~mL} /$ hour (6).

\section{Role of team members}

The thoracic surgeon and the assisting surgeon are standing on the anterior side of the patient wearing $3 \mathrm{D}$ glasses and looking at the $3 \mathrm{D}$ screen. It is important that the screen is large and at least $1.5 \mathrm{~m}$ away from the surgeons to get the maximal benefit of the 3D effect. Ergonomic is important to prevent back and neck injuries. Therefore, the screen should be placed straight opposite the surgeons $10 \mathrm{~cm}$ below their horizontal eye axis. The scrub nurse is standing on the opposite side of the patient with a separate screen in $2 \mathrm{D}$, since wearing $3 \mathrm{D}$ glasses impairs her overview of the instrument table. For VATS surgery, it is important to have a close cooperation with the anesthesiologist. Full relaxation of the patient is important to avoid elevation of the diaphragm during surgery. Correct placement of a double lumen tube is important to ensure sufficient lung isolation. The use of camera tubes allows for easy replacement in the case the surgeon displaces the double lumen tube due to manipulation of the main bronchus (7).

\section{Postoperative management}

The patient was extubated the operating theater and transferred to a postoperative recovery room staying overnight under observation. An enhanced recovery protocol is applied to all patients undergoing thoracic procedures in our department according to the recent guidelines from ESTS and the ERAS society (8). The chest drain was connected to a digital drainage device (Topaz+, Medela) and the suction level were set to $-2 \mathrm{cmH}_{2} \mathrm{O}$ (9). The patient was mobilized on the day of surgery. Pain was controlled using a multimodal opioid sparring regimen (6). The postoperative course was uneventful. The chest 
drain was removed the next morning and the patient was discharged on the second postoperative day. A visit to the outpatient clinic were scheduled two weeks after surgery and the patient were doing well and at 6 months follow-up the patient had not experienced any pulmonary infections.

\section{Tips, tricks and pitfalls}

(I) When dividing the feeding artery for a sequestration, it is recommended to dissect up to $2 \mathrm{~cm}$ of the artery and divide with it using an endostapler either tan or purple cartilage depending on the size of the artery. Care should be taken to have a long stump, otherwise the proximal end may slip into the abdomen and a potential stump insufficiency may bleed into the abdomen and not detected in the chest drain output or on chest X-ray.

(II) Due to the recurrent infections in intra lobar sequestrations, the hilar lymph nodes are inflammatory enlarged and sticky. Advanced energy tools are useful for this kind of dissection by dividing the small lymphatic vessels and keeping the field dry, improving the overview of the dissection.

(III) In segmentectomies it is often necessary to mobilize the hilar vessels and bronchi of the segment to be removed from the adjacent lung parenchyma in order to avoid stapling of the hilar structures of the segments that are preserved to avoid obstruction of the vessels and a potential risk of necrosis.

(IV) Intersegmental planes can be localized using different techniques. The classical technique is the inflation/ deflation technique. Inflation of the reaming part of the lung may obstruct the field of vision, especially in VATS. Newer techniques such as injection of indocyanine green and visualization with a near infrared camera may be easier to use for VATS surgery (10).

\section{Conclusions}

A left intra lobar sequestration in the lower lobe was managed safely with a lung sparring minimal invasive approach. The course was uneventful with a short inhospital stay. Identification of the intersegmental plane using indocyanine green and near infrared seems promising.

\section{Acknowledgments}

Funding: None.

\section{Footnote}

Provenance and Peer Review: This article was commissioned by the Guest Editor (Michel Gonzalez) for the series "VATS Segmentectomy" published in Fournal of Visualized Surgery. The article has undergone external peer review.

Reporting Checklist: The author has completed the CARE reporting checklist. Available at https://jovs.amegroups. com/article/view/10.21037/jovs-21-21/rc

Peer Review File: Available at https://jovs.amegroups.com/ article/view/10.21037/jovs-21-21/prf

Conflicts of Interest: The author has completed the ICMJE uniform disclosure form (available at https://jovs. amegroups.com/article/view/10.21037/jovs-21-21/coif). The series "VATS Segmentectomy" was commissioned by the editorial office without any funding or sponsorship. The author has received speaker fee from Medtronic outside the submitted work and has participated in Advisory Boards for AstraZeneca; and serves as an unpaid editorial board member of Fournal of Visualized Surgery from October 2020 to September 2022. The author has no other conflicts of interest to declare.

Ethical Statement: The author is accountable for all aspects of the work in ensuring that questions related to the accuracy or integrity of any part of the work are appropriately investigated and resolved. All procedures performed in this study were in accordance with the ethical standards of the institutional and/or national research committee(s) and with the Helsinki Declaration (as revised in 2013). Written informed consent was obtained from the patient for publication of this manuscript and the accompany images and video. A copy of the written consent is available for review by the editorial office of this journal.

Open Access Statement: This is an Open Access article distributed in accordance with the Creative Commons Attribution-NonCommercial-NoDerivs 4.0 International License (CC BY-NC-ND 4.0), which permits the noncommercial replication and distribution of the article with the strict proviso that no changes or edits are made and the original work is properly cited (including links to both the formal publication through the relevant DOI and the license). See: https://creativecommons.org/ licenses/by-nc-nd/4.0/. 


\section{References}

1. Pryce DM. Lower accessory pulmonary artery with intralobar sequestration of lung; a report of seven cases. J Pathol Bacteriol 1946;58:457-67.

2. Chakraborty RK, Modi P, Sharma S. Pulmonary Sequestration 2021 Mar 1. In: StatPearls [Internet]. Treasure Island (FL): StatPearls Publishing; 2021 Jan.

3. Traibi A, Seguin-Givelet A, Brian E, et al. Adult pulmonary intralobar sequestrations: changes in the surgical management. J Vis Surg 2018;4:62.

4. Hansen HJ, Petersen RH. Video-assisted thoracoscopic lobectomy using a standardized three-port anterior approach - The Copenhagen experience. Ann Cardiothorac Surg 2012;1:70-6.

5. Petersen RH, Hansen HJ. VATS segmentectomy utilizing the Copenhagen approach. Ann Cardiothorac Surg 2014;3:211-2.

6. Wildgaard K, Petersen RH, Hansen HJ, et al. Multimodal

doi: $10.21037 /$ jovs-21-21

Cite this article as: Petersen RH. Left basilar VATS segmentectomy for intra lobar pulmonary sequestration using indocyanine green for identification of the intersegmental plane: a case report. J Vis Surg 2023;9:15. analgesic treatment in video-assisted thoracic surgery lobectomy using an intraoperative intercostal catheter. Eur J Cardiothorac Surg 2012;41:1072-7.

7. Schuepbach R, Grande B, Camen G, et al. Intubation with VivaSight or conventional left-sided double-lumen tubes: a randomized trial. Can J Anaesth 2015;62:762-9.

8. Batchelor TJP, Rasburn NJ, Abdelnour-Berchtold E, et al. Guidelines for enhanced recovery after lung surgery: recommendations of the Enhanced Recovery After Surgery (ERAS®) Society and the European Society of Thoracic Surgeons (ESTS). Eur J Cardiothorac Surg 2019;55:91-115.

9. Holbek BL, Christensen M, Hansen HJ, et al. The effects of low suction on digital drainage devices after lobectomy using video-assisted thoracoscopic surgery: a randomized controlled trial. Eur J Cardiothorac Surg 2019;5 5:673-81.

10. Andolfi M, Potenza R, Seguin-Givelet A, et al. Identification of the intersegmental plane during thoracoscopic segmentectomy: state of the art. Interact Cardiovasc Thorac Surg 2020;30:329-36. 